\title{
Modernización ecológica, cambio tecnológico y globalización
}

\author{
Guillermo Foladori*
}

\begin{abstract}
This article discusses the trends of the environmental technological policies and their relationship to the process of globalisation. The history of the last thirty years and the trends of economical globalisation seem to show that the policies of control of emissions, i.e. "end of pipe technologies", are the main priority. At the same time, the transnational enterprises, supported by the growing commercial liberalisation, have a role with ever more importance in the national and international environmental policies. They exert pressure to include only those environmental problems of interest to developed countries in commercial treaties.
\end{abstract}

Keywords: sustainability, globalisation, nanotechnology.

\section{Resumen}

Este artículo muestra las tendencias de las políticas tecnológicas en materia ambiental y su relación con el proceso de globalización. La historia de los últimos 30 años y las tendencias de globalización de la economía parecen mostrar que las políticas de control de emisiones, end of pipe technologies, son las prioritarias. Al mismo tiempo, las corporaciones transnacionales apoyadas en la creciente liberalización del comercio tienen un papel cada vez más significativo en las políticas ambientales nacionales e internacionales, y presionan para que sean incorporados a los tratados de comercio sólo aquellos problemas del ambiente que interesan a los países desarrollados.

Palabras clave: sostenibilidad, globalización, nanotecnología.

\footnotetext{
*Universidad Autónoma de Zacatecas. Correo-e: fola@estudiosdeldesarrollo.net.
} 


\section{Introducción}

Las políticas de desarrollo sostenible se han orientado, en gran medida, hacia las modificaciones tecnológicas. Dentro de las alternativas tecnológicas pueden distinguirse dos procedimientos no necesariamente contrapuestos. Por un lado, el control de emisiones, que tiene como propósito corregir las emisiones contaminantes. Por otro, el control de fuentes, que intenta cambiar las tecnologías contaminantes por otras que no lo sean, lo que obliga, en muchos casos, a un cambio en el patrón energético. Cuando ambas políticas pasan de simples correcciones, al estilo de bomberos que apagan fuegos ocasionales, a formar parte de una programación global de la economía de un país, se habla de un proceso de modernización ecológica. ${ }^{1}$

Este artículo muestra las orientaciones de las políticas tecnológicas en materia ambiental y su relación con el proceso de globalización. La historia de los últimos 30 años y las tendencias de globalización de la economía parecen mostrar que las políticas de control de emisiones, llamadas comúnmente end of pipe technologies, son las prioritarias. Al mismo tiempo, las corporaciones transnacionales apoyadas en la creciente liberalización del comercio tienen un papel cada vez más significativo en las políticas nacionales e internacionales, $y$ presionan para que sean incorporados a los tratados de comercio sólo aquellos problemas ambientales de interés para los países desarrollados. Así, eventualmente, forman parte de los acuerdos comerciales las restricciones de actividades que agravan el calentamiento global, perjudican la biodiversidad o propagan la deforestación; mientras, sistemáticamente se dejan fuera de los acuerdos comerciales aquellas actividades que tienen efectos locales y no globales, como la contaminación de cursos de agua locales, el aire contaminado de las ciudades, la basura tóxica, los componentes químicos de alimentos nocivos para la salud o los asentamientos en zonas de riesgo. Con ello se obliga a los países del Tercer Mundo a asumir como problemas ambientales mundiales muchos que sólo son de interés para los países desarrollados, mientras se dejan de lado otros, de gravedad confirmada, en el ámbito regional o local.

\footnotetext{
${ }^{1}$ El término "modernización ecológica” debe atribuirse al sociólogo alemán Joseph Huber, quien comenzó a utilizarlo en 1982 (Brey, 1999).
} 


\section{Los enfoques de las emisiones y las fuentes de los problemas ambientales}

Hasta mediados de la década de los sesenta del siglo xx, el crecimiento económico era considerado la vía para el bienestar de la sociedad. En algunos casos surgían críticas, mostraban que tal crecimiento no conducía a una equidad o justicia social y que debía, por lo tanto, distinguirse el simple crecimiento económico del que mejora la distribución del producto social y que tiende hacia la equidad. A este último se le llamó desarrollo. Pero a partir de mediados de los sesenta, en los países desarrollados surge la preocupación de que el crecimiento, además de no solucionar los problemas de equidad y justicia social, podía provocar daños irreparables en el medio. Con ello el concepto de desarrollo también resultó afectado y dejaba de ser un buen sustituto del término crecimiento. La solución conceptual, luego de más de diez años de utilizar diversas palabras, fue desarrollo sostenible. ${ }^{2}$ Este concepto debía garantizar una mejoría de las condiciones sociales y también del ecosistema, que en definitiva es la fuente de cualquier economía. Si se lograban ambos objetivos, se estaría en la dirección adecuada para el logro de una mejor distribución de la riqueza, tanto de las presentes generaciones como de las futuras, que recibirían un ambiente igual o mejor que el actual.

Las políticas para encaminar el desarrollo hacia la sostenibilidad que afectaron la tecnología tuvieron, desde un comienzo, dos modalidades diferentes: las end of pipe (técnicas de control de emisiones) que trataron de solucionar las emisiones perjudiciales y las dirigidas a las fuentes u orígenes tecnológicos de los problemas ambientales, lo que en muchos casos implica el cambio completo de tecnología o del tipo de energía utilizado, o de ambos. Estos dos tipos de política no son contradictorios y pueden, en muchos casos, ser complementarios. Pero cualquiera de estos enfoques considera a la tecnología como causa principal de los problemas ambientales, de allí que un cambio tecnológico podría conducir a la sostenibilidad esperada. Con bases débiles se plantea la posibilidad de que las relaciones de producción capitalista o su 'estilo de vida' de creciente consumo suntuario puedan ser causa de los problemas ambientales, en cuyo caso la so-

\footnotetext{
${ }^{2}$ El concepto de desarrollo sostenible se internacionalizó a partir del "Informe Brundtland", Nuestro Futuro Común, un documento sobre la situación ambiental del mundo encomendado por la Organización de las Naciones Unidas a un grupo ad hoc y publicado en 1986.
} 
lución tecnológica, aunque parcial, no sería definitiva. Según algunos críticos, serían las propias relaciones sociales de producción capitalista las que conducen al deterioro del ambiente (Burkett, 1999 y Foster, 2000). Se afirma que el propósito de la producción capitalista es el lucro, y en la producción de mercancías es normal el daño ambiental porque el abaratamiento de los costos de producción, que significa mayor ganancia, es parte de la lógica capitalista; y dentro de la disminución de costos está la sobreexplotación de la naturaleza o la utilización de los espacios públicos como basurero privado. También se incrementa la ganancia si se aumenta la rotación del capital, y para que esto suceda es necesario que los productos tengan una vida útil corta, de manera que otros nuevos ocupen su lugar. Así, se impulsa la producción de más y más objetos cada vez menos útiles en términos de durabilidad, aunque puedan serlo en función inmediata; con ello se incrementa el consumo de recursos naturales y la basura. Por último, la propia tecnología también se produce con el fin de obtener ganancias, con lo cual sus efectos, que pueden ser externos, la abaratan y sólo benefician al empresario. De esta forma, los mecanismos rigurosos de seguridad contra elementos tóxicos no serán utilizados donde el costo de la vida humana sea barato, tampoco se solucionarán los efectos tóxicos de los residuos de agroquímicos. La tecnología capitalista es, por su origen como vehículo de lucro, un elemento diseñado independientemente del contexto (Burkett, 1999 y Foster, 2000). Esto podría explicar, aunque sólo en parte, que la aplicación de tecnología idéntica, de origen capitalista, en la ex Unión Soviética y en Cuba ocasionó efectos devastadores en el ambiente similares a los de países capitalistas (Commoner, 1992; Díaz Briquets y Pérez López, 2000). Según otros críticos, el estilo de vida capitalista tiende al consumo ilimitado y fomenta la producción infinita, que se contrapone directamente a un planeta con recursos finitos, asunto tampoco resuelto por las medidas tecnológicas (Daly, 1989).

Las políticas dirigidas para controlar las emisiones pueden ser vistas como contradictorias en relación con las dirigidas a las fuentes. A principios de los años setenta, Barry Commoner, conocido biólogo estadounidense y militante pacifista y ecologista, mostraba que las políticas dirigidas al control de las emisiones end of pipe technologies- no lograban su objetivo al menos por tres razones: a) ningún dispositivo de control es perfecto, pueden ocurrir fallas, como lo ha constatado la historia de los accidentes, tanto en los países capitalistas como en los socialistas; $b$ ) 
los dispositivos de control nunca alcanzan efectividad total, de manera que si alcanzan 90\%, el 10\% restante contamina la atmósfera, y el volumen absoluto puede crecer si aumenta la actividad de las industrias que cuenten con dichos dispositivos, y $c$ ) los dispositivos de control pierden eficacia con el uso. Es sabido que un auto nuevo, que rinde $15 \mathrm{~km}$ por litro de gasolina en el primer año, sólo recorre $12 \mathrm{~km}$ con un litro 10 años después. Por estas razones, Commoner se pronunciaba por cambiar radicalmente las tecnologías contaminantes: estaba en contra de las políticas de end of pipe y a favor de las que cambian la tecnología por otra no contaminante.

Una alternativa o complemento de las políticas dirigidas al control de las emisiones es la orientada a las fuentes. Suponen cambiar la tecnología, incluso el tipo de producto generado y la fuente energética. Pero estas políticas son mucho más difíciles de implantar, tanto por la resistencia de las industrias, que ya trabajan con determinadas tecnologías, como porque modificar las fuentes afecta los sistemas de energía y se provocaría una transformación muy importante en las bases técnicas y de infraestructura del país en cuestión. Esta modalidad conduce a una política de programación de la economía como un todo, que simultáneamente considere: $a$ ) el control de las emisiones, $b$ ) el control de los volúmenes de producción (dependiendo de los materiales y condiciones de producción), y c) la modificación de las fuentes energéticas y los procesos de producción y consumo. A esta alternativa se le llama "modernización ecológica" (Brey, 1999). El análisis ambiental estratégico (AAE) es un método participativo desarrollado con el fin de incorporar los problemas ambientales a los planes de desarrollo. En términos generales, el AAE tiene tres fases bien definidas. ${ }^{3}$ En la primera se realiza el diagnóstico de la situación de las funciones ambientales y el beneficio o perjuicio que los grupos de interés obtienen de ellas y en qué forma. La segunda fase implica la identificación de cómo esas funciones son impactadas, o bien, cuáles son las potencialidades que ofrecen. La tercera fase constituye la elaboración del plan ajustado al tamiz ambiental y los mecanismos de monitoreo (Kessler, 2003).

\footnotetext{
${ }^{3}$ El AAE es, en palabras de Dalal-Clayton y Sadler (1999: 1), “un proceso sistemático de evaluación de las consecuencias ambientales de políticas propuestas, planes o iniciativas de programas, con el propósito de asegurar que están plenamente incluidas y apropiadamente contempladas en las primeras etapas de las decisiones en igualdad de condiciones que las consideraciones económicas y sociales".
} 
No obstante, según los ecologistas radicales, ninguna alternativa exclusivamente tecnológica ayudará en la sostenibilidad, sea de end of pipe o de cambio tecnológico. Primero, porque los cambios tecnológicos generalmente implican costos mayores que las empresas no están dispuestas a asumir, así no llegan a implantarse. Segundo, porque la tecnología no solucionará los problemas de injusticia y pobreza social. Tercero, porque la tecnología siempre acarrea resultados imprevistos; y cuarto, porque no se frena el crecimiento ilimitado que entraría en contradicción con los supuestos recursos limitados del planeta (Brey, 1999).

Aunque la modernización ecológica considere la corrección de las fuentes y las emisiones, las diferencias en las políticas entre unas y otras son significativas; en el caso de las emisiones, son de corto plazo; en el de las fuentes, están dirigidas a los cambios tecnológicos estructurales y de largo plazo. Desde el punto de vista político-institucional, la diferencia es radical. Para establecer políticas dirigidas a las emisiones basta un ministerio del medio ambiente que regule y fiscalice. Para poner en marcha políticas dirigidas a las fuentes, el ministerio del ambiente tendrá que convertirse en el eje del ministerio de planificación, ya que la forma de producir debe prever el contexto global de la economía. Además existe una diferencia económica de peso. Los mecanismos dirigidos a las emisiones pueden, en muchos casos, orientarse y regularse mediante lo conocido como "instrumentos de mercado" (cuotas negociables de contaminación, impuestos a las emisiones contaminantes, sistemas de reembolso de envases, etc.), lo cual significa costos bajos de regulación para el Estado, y un mecanismo que permite la competencia y favorece a los sectores de vanguardia tecnológica. Por otra parte, una programación general de la economía hacia el desarrollo sostenible que ponga énfasis en las fuentes requiere un Estado mucho más fuerte que enfrente directamente a los sectores nacionales e internacionales. Además, para los gobiernos, desarrollo y ambiente se presentan muchas veces como competidores por los escasos recursos del presupuesto público, y en los países del Tercer Mundo, donde los problemas sociales son más acuciantes, la situación se agudiza y se menosprecia el ambiente en favor del supuesto desarrollo. No es casual, por tanto, que en los ámbitos nacional e internacional la mayoría de los esfuerzos hacia la sostenibilidad provenga de políticas end of pipe o dirigidas a las emisiones. 
La tendencia hacia las políticas de control de emisiones presenta contratendencias. No todo proceso económico-social es mecánico ni unilateral. Cuando ocurren accidentes o desastres que afectan la vida humana, la población se rebela contra las empresas que lo ocasionaron, contra las ramas de la producción y las corporaciones transnacionales. Los movimientos ambientalistas y ecologistas retoman esta indignación social para fortalecer sus organizaciones y se colocan al frente de las reivindicaciones. La mayoría de las veces hay cierta distancia entre las causas objetivas de los problemas y los temores que despiertan en la población. De allí que los accidentes que ocurren en países del Tercer Mundo sean vistos en Estados Unidos como modelo de lo que podría acaecer en su propio territorio, aunque los mecanismos de seguridad y la tecnología sean más eficientes y, por lo tanto, los riesgos sean mucho menores. Frente a esta situación, la presión de la sociedad mediante organizaciones ambientalistas y ecologistas obligó a los gobiernos a cambiar la orientación de las reglamentaciones, se pasó de las políticas de control de emisiones a las que controlan las fuentes. En Estados Unidos, por ejemplo, la United States Environmental Protection Agency (EPA), que surgió en 1972, introdujo la prevención de la contaminación en 1989. Esto significaba reducir las emisiones en lugar de filtrarlas; también suponía limitar o minimizar los desperdicios, sustituir fuentes de energía y materiales, modificar procesos y garantizar el recicle. El Pollution Prevention Act que comenzó a regir en esa nación forzó en 1990 a las empresas a entregar información sobre los esfuerzos para reducir los desperdicios (García Johnson, 2000: 67). El cambio del control de las emisiones por el de las fuentes, que en primera instancia puede incrementar aún más los costos de producción tiene, una vez logrado el cambio tecnológico necesario, un efecto inverso y abarata los costos. La clave está en la utilización de los materiales eficientemente y en la reducción del desperdicio, lo que disminuirá los costos de producción. Mientras que en el pasado el concepto de productividad del trabajo era la llave para el progreso, hoy se habla cada vez más de productividad de los recursos, lo cual no significa producir menos ni con menor eficiencia, por el contrario, se producirá más y mejor utilizando los recursos eficientemente y generando, por lo tanto, menos desperdicio (Von Weizsäcker et al., 1997). 
2. El papel de la globalización y el comercio internacional en el ambiente de los países del Tercer Mundo

El movimiento de capitales en el ámbito internacional tiene efectos contradictorios en la situación ambiental de los países del Tercer Mundo. Veamos las principales tendencias.

\subsection{El capital internacional busca entre los países bajos costos}

Entre los costos bajos están los salarios mínimos, recursos naturales de fácil acceso y reglamentaciones muy flexibles para la emisión de desechos. En cualquier caso, siempre se perjudica al ambiente en esta migración de empresas. En un comunicado interno del Banco Mundial, el entonces vicepresidente de esa organización, Larry Summers, argumentaba las ventajas económicas de trasladar industrias contaminantes del Primer al Tercer Mundo. Anotaba que: a) los salarios eran menores en los países del Tercer Mundo, por lo tanto los seguros de vida, que se calculan de acuerdo con el monto de los salarios, también lo son. De allí que las indemnizaciones por muerte, enfermedad y lesiones sean más baratas; $b$ ) los países ricos valoran más su ambiente y, por lo tanto, tienen mayores restricciones y costos para inversiones contaminantes; $c$ ) los países pobres están menos contaminados, por lo cual la capacidad del ecosistema para absorber la contaminación es mayor. Ejemplos hay muchos. Uno de los más célebres fue la explosión de la planta de la Union Carbide en Bhopal, India, en diciembre de 1984, que mató entre 2,000 y 5,000 personas, dejó con afecciones pulmonares permanentes a otras 86,000 y los reclamos fueron 600,000. La planta de Bhopal tenía condiciones menores de seguridad que su hermana de West Virginia, Estados Unidos. Los equipos de detección de vapores eran de calidad inferior y los sistemas de emergencia no eran adecuados para el tamaño de las instalaciones ni operaban automáticamente (Sem, 1995 y Karliner, 1997). Pero para la lógica económica, es más rentable pagar seguros de vida de 100,000 dólares, que es la media en los países del Tercer Mundo, que 1'000,000 de dólares como en los países del Primer Mundo. Por lo demás, la ideología capitalista convalida el desastre químico de Bhopal como resultado de la libertad de vivir y trabajar. La revista Time, de ese mismo mes, publicó en su página 20: 
Los ciudadanos de Bhopal vivían cerca de la planta de Union Carbide porque querían vivir allí. La planta proporcionaba puestos de trabajo, el plaguicida más alimentos. Bhopal fue una parábola moderna de los riesgos y recompensas originalmente engendrados por la Revolución Industrial [...] No hay forma de evitar este riesgo, e intentarlo no sirve de nada; sólo confiamos en que los dioses de las máquinas nos den mucho más de lo que nos quitan (en Commoner, 1992: 63).

Pero esta migración de las industrias contaminantes a los países del Tercer Mundo tiende a homogeneizar las reglamentaciones una vez que los accidentes, como el de Bhopal, aumentan el grado de conciencia de la población y el número de movimientos ambientalistas y ecologistas que terminan por presionar a los gobiernos.

Ahora bien, es interesante notar que los estudios realizados por el Centro de Corporaciones Transnacionales de las Naciones Unidas durante los años setenta y ochenta mostraron que sólo unas pocas empresas emigraron de Estados Unidos hacia el Tercer Mundo debido a las ventajas económicas derivadas de una legislación menos exigente en materia ambiental. La causa principal fueron los salarios más baratos (García Johnson, 2000: 87). Esto muestra una vez más la falsedad de dividir los problemas ambientales de los sociales. Cuando las industrias son de riesgo, uno de los costos principales son los seguros humanos. El caso de Bhopal es elocuente, es suficiente con que los salarios sean más baratos para que estemos frente a una ventaja ambiental disfrazada de social. Mediante una encuesta realizada en 1991 por Rappaport y Flaherty entre casi 100 corporaciones transnacionales, se encontró que no se aplicaban los mismos controles fuera de los países sede (García Johnson, 2000: 89), lo que muestra que las ventajas económicas ambientales existen, aunque no sean explícitas.

\subsection{La liberalización comercial es utilizada como regulador de los intereses ambientales}

La globalización ha redimensionado el papel del mercado mundial y subsume el de los Estados nacionales. La Organización Mundial del Comercio (OMC) tiene como propósito establecer reglas para el comercio mundial, eliminar tarifas y otras barreras con el objetivo de liberar el acceso a los mercados. Se trata de evaluar el comercio de los países para permitirles la entrada a la organización o establecer sanciones. Este es un argumento co- 
mercial para que los Estados miembros no reconozcan acuerdos bi o multilaterales sobre el ambiente. Así se favorece a las grandes corporaciones transnacionales y se debilita el poder de los Estados nacionales en la decisión sobre su ambiente. La OMC se ha convertido en 'foro' para la discusión de problemas ambientales. Por ejemplo, Estados Unidos utilizó criterios ambientales en contra de la importación de carne y productos de ganado criado con hormonas en la Comunidad Europea durante 1989; al mismo tiempo presiona por la apertura de los mercados de transgénicos. En suma, utiliza criterios 'ecológicos', según los intereses comerciales, en favor o en contra del ambiente. Estos y muchos otros casos documentados muestran que la desregulación de la economía lleva a que las corporaciones transnacionales tengan mayor poder sobre los gobiernos nacionales y coloca los intereses comerciales sobre las reglamentaciones ambientales (Hines, 2000).

\subsection{Las preocupaciones ambientales de los países desarrollados son diferentes de las de los países del Tercer Mundo}

Existe diferencia entre la contaminación que ocurre en los países del Primer Mundo y en los del Tercer Mundo. Debido a restricciones ambientales mayores impuestas desde los setenta, los países desarrollados viven en ambientes menos contaminados y están preocupados por los problemas o efectos globales de largo alcance, como el calentamiento global, la perforación en la capa de ozono o la pérdida de la biodiversidad. Esto hace que no presten atención a los problemas ambientales locales generados por las corporaciones transnacionales en los países subdesarrollados, que afectan de manera inmediata a sus habitantes. Los problemas por basura tóxica, los efectos de los químicos utilizados en la agricultura y la contaminación de los cursos de agua y del aire de las ciudades son menospreciados por el Primer Mundo. Esto puede constatarse en los acuerdos de comercio internacional, donde con dificultad se incluyen cláusulas que defienden el ambiente y sólo se refieren al calentamiento global o a la biodiversidad, como fue el caso del Tratado de Libre Comercio de América del Norte entre Canadá, Estados Unidos y México. Esta presión de los países desarrollados por centrar las discusiones y acuerdos en los problemas ambientales 'globales', una vez que han superado relativamente los locales o regionales, ha propicia- 
do una atmósfera de intereses comunes entre los habitantes del mundo. Lo que no se dice es que las incertidumbres científicas sobre los problemas del calentamiento global o del agujero en la capa de ozono son mayores que las certezas; al mismo tiempo, gran parte del complejo militar que hasta los años ochenta tenía su mira puesta en la investigación sobre el átomo derivó hacia las investigaciones meteorológicas y del cambio climático (Le Bras, 1997; Lenoir, 1995; Tommasino y Foladori, 2001). Mientras, no resulta claro para quiénes tendrá efectos negativos o positivos el calentamiento global o el adelgazamiento de la capa de ozono. Están totalmente demostradas las consecuencias de los productos orgánicos clorados fabricados por la industria química para la salud de los seres humanos y otros seres vivos, así como los riesgos de la energía nuclear, también de la contaminación atmosférica con detritos humanos resultado de la falta de saneamiento en la mayoría de las ciudades del Tercer Mundo. Asimismo, los países ricos sufren el exceso de dióxido de carbono en la atmósfera, que tiene efectos dudosos y a largo plazo; los del Tercer Mundo soportan la contaminación por sulfatos, nitratos, plomo y mercurio, que tienen efectos inmediatos y ampliamente confirmados. Por otra parte, la pobreza y el crecimiento de la población sólo son vistos como problemas ambientales por el Primer Mundo en la medida que ambos factores presionan sobre los recursos o cuando las prácticas agrícolas tradicionales de roza y quema perjudican los ecosistemas y aumentan el calentamiento global; esto es, los países del Tercer Mundo son vistos sólo como puente para los problemas ambientales considerados centrales por el Primer Mundo (Foladori y Tommasino, 2000).

\subsection{Existen contratendencias favorables al ambiente derivadas de la liberalización del comercio mundial}

Aunque en términos generales puede decirse que las corporaciones transnacionales sólo invierten en tecnologías limpias cuando les son exigidas, una vez que lo logran y superan a sus competidores, pagan con la misma moneda. Presionan para que se establezcan acuerdos internacionales de niveles de contaminación, promueven normas de calidad ambiental, como la Isso 14000 , y cabildean en otros países para que los gobiernos locales obliguen a las empresas a adaptarse a los patrones de calidad ambiental de las corporaciones transnacionales. Así compiten ventajosamente, y lo que en un momento fue un gran costo se convierte en 
ganancia extraordinaria y en un mayor monopolio. En la industria química, por ejemplo, ha habido un cambio significativo a partir de los años noventa como resultado de los graves accidentes de los ochenta. La política denominada Responsible Care, promovida por la Asociación de Industrias Químicas de Canadá y Estados Unidos luego del desastre de Bohpal, mejoró la seguridad de los trabajadores, la salud y el ambiente. Esta política se aplica desde principios de los años noventa en todos los países sede de las corporaciones transnacionales de la industria química. Con ello, están exportando 'ambientalismo' a los países del Tercer Mundo, además presionan para que los criterios de seguridad ambiental y su legislación se eleven. Un estudio publicado recientemente sobre las corporaciones transnacionales de la industria química en México y Brasil muestra que se introdujeron las medidas de control de contaminación Responsable Care antes que las reclamasen los movimientos ambientalistas (García Johnson, 2000).

\subsection{La globalización obliga a los Estados nacionales a buscar recursos en el fluctuante capital financiero transnacional}

Para atraer inversiones extranjeras, los gobiernos nacionales y regionales compiten por facilitar condiciones de inversión. Esto supone, la mayoría de las veces, condiciones blandas para el pago de impuestos, otorgamiento de áreas geográficas subvaluadas o gratis, liberar restricciones ambientales para permitir asentamientos industriales e infraestructura en zonas prohibidas o de riesgo ambiental. Con ello, los gobiernos nacionales y regionales se vuelven cada vez más débiles frente a las inversiones de las corporaciones transnacionales y los resultados, entre otros, son la construcción de hoteles de cinco estrellas en las playas, o de edificios elevadísimos en zonas residenciales y consecuente pérdida de calidad ambiental, y en la instalación de industrias en zonas de inundación o riesgo ambiental (Sowers et al., 2000; Torres y Costa, 2000).

\section{La revolución de la nanotecnología y posibles perspectivas}

En los años recientes ha cristalizado una nueva revolución tecnológica que tendrá impactos significativos en el ambiente y el comercio mundial. Se trata de la nanotecnología. Consiste en la manipulación de los átomos para formar productos. Desde el 
punto de vista tecnológico es una revolución por dos razones básicas. Primera: se trata de construir de lo más pequeño a lo más grande (bottom-up) en lugar de comenzar por la materia prima en gran escala, extraída tal cual existe en la naturaleza y reducirla al tamaño de los objetos de uso, como se hace hasta la fecha. Segunda: porque en el nivel atómico no hay diferencia entre materia biótica y abiótica, de manera que pueden aplicarse procedimientos de reproducción biológicos a los procesos materiales.

Según los apologistas de la nanotecnología, sus aplicaciones serán ampliamente benéficas para el medio. El proceso de producción bottom-up eliminará el desperdicio del proceso productivo; la producción a partir de los elementos químicos básicos volverá superflua la dependencia de los recursos naturales, que ya no serán demandados. La aplicación en el área de la energía permitirá utilizar energías limpias en gran escala. En la agricultura, los nanosensores podrán reducir sustancialmente el riego, el desperdicio de nutrientes y prácticamente de todos los herbicidas, funguicidas y pesticidas. También podrá potabilizarse agua a costos muy bajos y los catalizadores descontaminarán el aire. En el área de la salud los efectos serán impresionantes: permitirán que los fármacos lleguen directamente a las células que los requieran. Estos serían algunos de los resultados positivos. ${ }^{4}$

Aunque se trata de una revolución tecnológica en ciernes y resulta difícil predecir a qué ramas de la producción se expandirá, el hecho es que ya está rediseñando la discusión ambientalista. En primer lugar, la diferencia en las tecnologías de control de las fuentes o de las emisiones, end of pipe, se vuelve trivial a la luz de la nanotecnología. Ésta controlaría las fuentes y, prácticamente, no habría emisiones. En segundo, la discusión acerca de si son más graves los problemas de contaminación o los de depredación también se vuelve superflua, ya que la nanotecnología está demostrando su capacidad para intervenir en la sustitución de materiales y en la elaboración de catalizadores y sensores que permiten revertir drásticamente la contaminación de la atmósfera, los cursos de agua y el suelo mediante nanopartículas compuestas por hongos y bacterias 'limpiadoras'.

Esta visión resulta demasiado optimista para algunos. Debido a la presión de algunas organizaciones no gubernamentales (ONG), entre las que destaca el grupo Erosión, Tecnología y Concentra-

${ }^{4}$ El área militar es la que mayor financiamiento público recibe en Estados Unidos (más de un tercio) para la investigación en nanotecnología y será, probablemente, la mayor beneficiaria de sus productos. 
ción (ETC), y diarios internacionales, ${ }^{5}$ desde el año 2000 instituciones oficiales de algunos gobiernos, como los de Estados Unidos y de Reino Unido, comenzaron a promover investigaciones sobre los posibles impactos de la nanotecnología en el medio y la salud. Una vez más en la historia de la tecnología, las soluciones no vienen solas, y los efectos secundarios, en el comienzo imprevisibles, deben ser evaluados.

Aún son pocos los estudios sobre el tema, pero los indicios no son del todo halagüeños. Nanotubos de carbono instalados en la traquea o pulmones de ratas tienden a formar nódulos, posiblemente como consecuencia de la reacción del organismo a cuerpos extraños (Lam et al., 2004; Warheit et al., 2004). También se ha descubierto que las nanopartículas producen daño cerebral en peces (Christiansen y Andersen, 2004). Resulta impredecible qué pueda suceder en el ambiente con las partículas autorreplicables. El grupo ETC habla de la "plaga verde" para referirse a la posibilidad de un proceso incontrolable de reproducción de nanopartículas (ETC, 2003), por lo cual llamó a una moratoria en su investigación y producción.

Se habla poco de los alcances de la nanotecnología en las relaciones sociales, no se analiza más allá de las consecuencias para la salud o el ambiente. Es posible que los productos de la industria tradicional no puedan competir con aquellas que tengan incorporados nanosensores, nanodistribuidores o nanocatalizadores, etc. Esto provocaría la ruina de economías masivas, sobre todo en los países más atrasados, cuyas secuelas serían los aumentos del desempleo y la violencia. También está el problema de las regulaciones para nanoproductos; actualmente no las hay en ningún país; incluso las corporaciones multinacionales que incorporan nanopartículas a sus productos argumentan que no tienen nada nuevo y, por lo tanto, no deben someterse a criterios de toxicidad. Pero, al mismo tiempo, esas empresas luchan por patentar sus productos y afirman que la combinación a escala atómica que realizan no se encuentra en la naturaleza. De manera que, como ocurre con muchos productos tóxicos, los países más pobres se convertirán en los consumidores de productos de riesgo desconocido.

Ya existe competencia por el posicionamiento internacional en nanotecnología. Estados Unidos, Reino Unido, China, Japón,

${ }^{5}$ El New York Times, por ejemplo, publicó varios artículos del periodista B. J. Feder sobre el tema (New York Times, 03/02/2003, 14/04/2003, 19/05/2003, 07/07/2003, 03/11/2003), The Guardian hizo otro tanto (28/04/2003, 30/07/2003, 09/01/2004). 
Corea del Sur, Taiwán e Israel son algunos de los más entusiastas, pero prácticamente todas las grandes corporaciones multinacionales apuestan fuerte por esta tecnología.

\section{Conclusiones}

Para facilitar la sistematización del análisis presentado se enumeran las conclusiones principales.

1. El origen de los cambios en el desarrollo sostenible efectuados desde fines de los años sesenta es resultado de los movimientos de la población organizada en grupos ambientalistas, ecologistas, pacifistas, etc. Los cambios en el desarrollo sostenible realizados desde principios de los años setenta fueron básicamente técnicos y no tocaron las relaciones sociales de producción capitalistas ni el estilo de vida consumista. Esto supone la desvinculación de las relaciones sociales con la tecnología. La revolución nanotecnológica no escapa de esta disyuntiva, ya que las corporaciones transnacionales son las principales productoras e interesadas en la divulgación de estos productos, aun con los posibles efectos de incremento de la desigualdad en el mundo.

2. En la vía técnica hacia la sostenibilidad pueden distinguirse dos caminos. Por un lado, el que enfatiza el control de las emisiones colocando filtros o mejorando el destino de los desperdicios. Por otro lado, el de las fuentes, que requiere modificaciones más profundas en la tecnología y en muchos casos la sustitución de las fuentes energéticas y de materias primas. Este último camino es notoriamente más radical que el primero. Cuando un país pone en marcha un programa de desarrollo sostenible que pretende encauzar todos los movimientos económicos, se habla de un proceso de modernización ecológica. Tal vez el ejemplo más claro de dicha estrategia sea el Plan Nacional para una Política Ambiental del gobierno holandés (Nationaal Milieubeleids Plan, NMP) de 1989. El análisis ambiental estratégico y la participación son los mecanismos para conseguirlo. Pero esta disyuntiva entre regular la fuente o las emisiones, entre controlar 'desde arriba' o establecer procesos participativos, puede tener un cambio radical mediante procesos nanotecnológicos de producción de energía, de sustitución de materias primas y de limpieza del ambiente. Estamos frente a una salida técnica que podría colocar a ciertas corporaciones transnacionales a la vanguardia del ambientalismo.

3. La globalización y el peso cada vez mayor de las corporaciones transnacionales tiene efectos contradictorios sobre el 
medio. Por un lado, hay una tendencia a reubicar las industrias contaminantes y de riesgo en los países del Tercer Mundo, donde hay reglamentaciones ambientales menos rígidas y salarios más bajos. Eso supone el abaratamiento de costos y la creciente diferencia entre los países ambientalmente limpios (Primer Mundo) y los cada vez más contaminados y degradados (Tercer Mundo). Por otro lado, como resultado de los movimientos ecologistas y ambientalistas, se tiende a la homogeneización de las reglamentaciones ambientales. Para las empresas con tecnología de punta, la mejora en los patrones de calidad y seguridad ambiental y salud se convierte en un arma poderosa para desplazar del mercado a empresas más atrasadas y así aumentar su monopolio. Por eso, en algunos casos, las corporaciones transnacionales juegan el papel de exportadoras de ambientalismo y obligan a otros países a que adopten medidas de reglamentación ambiental y control que no tenían antes (García Johnson, 2000).

4. Lo que el capitalismo no logra resolver aun con las formas más avanzadas de modernización ecológica ni con la aplicación de la nanotecnología al deterioro ambiental son dos problemas centrales de la sostenibilidad. Por un lado, el desempleo. Las ventas de las 200 principales corporaciones transnacionales del mundo exceden las de todos los países del mundo con exclusión de los nueve más grandes, pero emplean sólo a 19 millones de trabajadores, lo que significa menos de $0.75 \%$ de los trabajadores del mundo. Aún más, entre 1993 y 1995 las principales 100 corporaciones transnacionales incrementaron sus inversiones $25 \%$ pero, durante el mismo periodo, recortaron $4 \%$ de su fuerza de trabajo (Hines, 2000: 72). La dinámica capitalista no puede evitar el aumento la pobreza y la diferenciación social y, con ello, incrementar el acceso desigual al ambiente, por lo cual las mejoras ambientales derivadas de tecnologías verdes o limpias se convierten en ventajas para menos personas y países, y para las futuras generaciones. Por otro lado, los resultados imprevistos del uso de tecnologías cada vez más complejas generan el principio de precaución que cada vez más se impone como criterio de seguridad; proclama que la tecnología se aplique hasta que demuestre ser inofensiva para el ambiente y está basado en información científica controlada por las mismas corporaciones transnacionales que dirigen a institutos y laboratorios de investigación directa o indirectamente a través de los subsidios a las universidades. Lo que hoy en día es una certeza mañana puede presentar grandes incerti- 
dumbres. La “plaga verde”, que eventualmente podría surgir con la nanotecnología, no debe descartarse.

\section{Bibliografía}

Brey, Philip (1999), "Sustainable Technology and the Limits of Ecological Modernization”, Ludus Vitalis, México, VII (12): 153-167.

Burkett, Paul (1999), Marx and Nature. A Red and Green Perspective, St. Martin's Press, Nueva York.

Christiansen, Kim y Per Dannemand Andersen (2004), Background Paper on Environmental and Risk Aspects of $\mathrm{Na}$ notechnology, Ministry of Science, Technology and Innovation, Copenhague.

Commoner, Barry (1992), En paz con el planeta, Crítica-Drakontos, Barcelona.

Dalal Clayton, Barry y Barry Sadler (1999), "Strategic Environmental Assessment: A Rapidly Evolving Approach”, Environmental Planning Issues, 18, International Institute for Environment and Development.

Daly, Herman (1989), "Introducción a la economía en estado estacionario", en Herman Daly (comp.), Economía, ecología, ética. Ensayos hacia una economía en estado estacionario, FCE, México.

Díaz Briquets, Sergio y Jorge Pérez López (2000), Conquering Nature. The Environmental Legacy of Socialism in Cuba, University of Pittsburgh Press, Pittsburgh.

ETC Group (2003), The Big Down: Atomtech-Technologies Converging at the Nano-Scal, 30 de enero, Winnipeg.

Foladori, Guillermo y Humberto Tommasino (2000), “Controversias sobre sustentabilidad”, Ambios, 1, Montevideo.

Foster, John Bellamy (2000), Marx's Ecology, Montlhy Review Press, Nueva York. 
García Johnson, Ronie (2000), Exporting Environmentalism. U.s. Multinational Chemical Corporations in Brazil and Mexico, The MIT Press, Cambridge, Massachusetts y Londres.

Hines, Colin (2000), Localization. A Global Manifesto, Earthscan, Londres.

Karliner, Joshua (1997), The Corporate Planet. Ecology and Politics in the Age of Globalization, Sierra Club Books, San Francisco.

Kessler, Jan Joost (2003), "Strategic Environmental Analysis (SEAN). Short Version", AIDEnvironment (Advice and Research for Development and Environment), http://www.aiden vironment.org, 8 de octubre de 2004.

Lam, Chiu-Wing, John T. James, Richard McCluskey y Robert Hunter (2004), "Pulmonary Toxicity of Single-Tall Carbon Nanotubes in Mice 7 and 90 Days after Intratracheal Instillation", Toxicological Sciences, 77: 126-134.

Le Bras, Hervé (1997), Los límites del planeta. Mitos de la naturaleza y de la población, Ariel Geografía, Barcelona.

Lenoir, Yves (1995), A verdade sobre o efeito estufa. Dossier de una manipulação planetária, Caminho da Ciência, Lisboa.

Sem, Partha (1995), "Environmental Policies and North-South Trade: A Selected Survey of the Issues”, en: Venkataraman Bhaskar y Andrew Glyn (eds.), The North, the South and the Environment. Ecological Constraints and the Global Economy, St. Martin's Press, Nueva York.

Sowers, Jeannie, Atul Kohli y George Sørensen (2000), "States and Sovereignty: Introduction", en Pamela S. Chasek (ed.), The Global Environment in the Twenty-First Century: Prospects for International Cooperation, The United Nations University, Tokio, Nueva York, París.

Tommasino, Humberto y Guillermo Foladori (2001), “(In) certezas sobre la crisis ambiental”, Ambiente \& Sociedade, Nepam-Unicamp, São Paulo, IV (8): 49-68. 
Torres, Haroldo y Heloisa Costa (orgs.) (2000), População e Meio Ambiente. Debates e Desafios, Editora SEnAC, São Paulo.

Von Weizsäcker, Ernest V., Amory B. Lovins y L. Hunter Lovnis (1997), Factor Four: Doubling Wealth, Halving Resource Use, Earthscan, Londres.

Warheit, David B., B. R. Laurence, K. L. Reed, D. H. Roach, G. A. M. Reynolds y T. R. Webb (2004), "Comparative Pulmonary Toxicity Assessment of Single-Wall Carbon Nanotubes in Rats", Toxicology Sciences, 77 (1): 117-125.

Enviado: 30 de abril de 2004. Aceptado: 7 de febrero de 2005.

Guillermo Foladori es doctor en economía por la Universidad Nacional Autónoma de México. Fue profesor visitante en el Doctorado en Medio Ambiente y Desarrollo en la Universidad Federal de Paraná (Brasil), en la Universidad de Columbia (Estados Unidos) y actualmente se desempeña como profesor en el Doctorado en Estudios del Desarrollo de la Universidad Autónoma de Zacatecas. Especialista en temas de ambiente, salud y en nanotecnología. Autor de Controversias sobre sustentabilidad. La coevolución sociedad-naturaleza (Miguel Ángel Porrúa Editores, México, 2001), coeditor con Naina Pierri de ¿̇Sustentabilidad? Desacuerdos sobre el desarrollo sustentable (Miguel Ángel Porrúa Editores, México, 2005, en prensa), y decenas de artículos en diversas revistas. 